\title{
Clinical Profile of Mortality and Treatment Profile of Survival in Patients with COVID-19 Pneumonia Admitted to Dubai Hospital
}

\author{
Rashid Nadeem ${ }^{a} \quad$ Ishma Aijazi ${ }^{b} \quad$ Ashraf Elhoufi $^{a} \quad$ Crystal Danthic \\ Nouha Azazad ${ }^{d}$ Mohd Kafeel Khan ${ }^{e}$ Mohannad Alheraki ${ }^{b}$ \\ Muhammed Jamshaid ${ }^{\mathrm{b}}$ Asadullah Nawazani $^{\mathrm{b}}$ Sadia Tariq ${ }^{\mathrm{b}}$ \\ Sobia Siddiq ${ }^{b}$ Muzammil Hafeez $^{f}$ Mukesh Kumar $^{\mathrm{e}}$ Islam Bon $^{\mathrm{a}}$ \\ Ahmed Elsousi $^{a} \quad$ Lamiaa Salama $^{a}$ Sahish Kamat ${ }^{a}$ Rami Abdalla ${ }^{a}$ \\ antensive Care Unit, Dubai Hospital, Dubai, UAE; ${ }^{b}$ Department of Internal Medicine, Dubai Hospital, Dubai, UAE; \\ 'Resident, Department of Pediatrics, Dubai Hospital, Dubai, UAE; ${ }^{d}$ Resident, Department of Internal Medicine, \\ Dubai Hospital, Dubai, UAE; ${ }^{e}$ Pediatric Intensive Care Unit, Dubai Hospital, Dubai, UAE; ${ }^{f}$ Neonatal Intensive Care \\ Unit, Dubai Hospital, Dubai, UAE
}

\section{Keywords}

COVID-19 · Corona $\cdot$ SARS-CoV-2 $\cdot$ Clinical profile $\cdot$ Steroids . Outcomes $\cdot$ Chloroquine

\begin{abstract}
Background: Most COVID-19 studies conclude old age and coexisting illnesses as mortality determinants owing to different populations or methodologies, or omitting factors affecting outcomes. Methods: We analyzed COVID-19 patients' data $(N=391)$ of Dubai Hospital between January 1, 2020 and June 30, 2020. Results: Only 19 patients (4.8\%) were UAE nationals, while 372 (95.2\%) were expatriates. Median age was 48 (interquartile range, $40-56$ ) years; $22 \%$ were $<40$ years, and only $16.6 \%$ were female. Cough was the most common symptom $(78.7 \%)$, fever was $77.4 \%$, and gastrointestinal symptoms were least common (13.8\%). Approximately $95 \%$ had elevated C-reactive protein (CRP) and D-dimers (79\%), lymphocytopenia $47.3 \%$, and thrombocytopenia $13.8 \%$. Mortality was $30 \%$ for the total sample and $50 \%$ in ICU patients. ICU patients were older than non-ICU (age; $49.6 \pm 10.9$ vs. $46.7 \pm 12.7$ years, $p=0.04$ ). Eighty-five percent of ICU pa-
\end{abstract}

karger@karger.com www.karger.com/dmj

Karger!"
(C) 2021 The Author(s)

Published by S. Karger AG, Base

This is an Open Access article licensed under the Creative Common Attribution-NonCommercial-4.0 International License (CC BY-NC) (http://www.karger.com/Services/OpenAccessLicense), applicable to the online version of the article only. Usage and distribution for commercial purposes requires written permission. tients required invasive mechanical ventilation, $78 \%$ vasopressors, $88 \%$ sedation, $84 \%$ muscle paralysis, while none require any of these in the medical group. Survivors had fewer patients with sedatives $(p=0.01)$. The median length of stay in the hospital was 19 days, ICU stays 14 days, and ventilator 11 days. The Mann-Whitney test showed that survivors spent more days in the ICU (median [IQR] 18 [6.5-29.5] vs. 11 [4-18], $p$ value 0.003 ) and the hospital (32 [14.5-49.5] vs. 14 [7-21], $p$ value 0.001 ) than nonsurvivors. Ferritin and D-dimers were higher in nonsurvivors, but CRP was lower in nonsurvivors (ferritin (ng/mL) median (IQR) 1,434 (661.5-2206.5) versus $1,362(630-2,094), p$ value $=0.017$, CRP $(\mathrm{mg} / \mathrm{L}) 118.7(53.4-$ $184)$ versus $134.9(66.5-203.2), p$ value 0.001 and $D$-dimer $(\mu \mathrm{g} / \mathrm{mL}) 1.54(0-3.13)$ versus $1.09(0-2.51), p$ value $=0.001)$. Multiple logistic regression analysis determined age, fever on admission, use of oxygen, mechanical ventilation, and steroids as predictors of survival. Conclusions: COVID-19 patients were young males with pre-existing conditions. Ferritin, CRP, and D-dimers were higher in nonsurvivors. Treatment with chloroquine, antivirals, and anticoagulation was not different between survivors and nonsurvivors. Steroid use was a survival predictor.

(c) 2021 The Author(s)

Published by S. Karger AG, Basel
Correspondence to:

Rashid Nadeem, nadeem.dr@gmail.com 


\section{Introduction}

COVID-19 is a novel beta coronavirus, first identified in Wuhan city, China, with a genetic makeup 96\% identical to a bat coronavirus $[1,2]$. Epidemiologic investigations and contact tracing of initial cases reveal that the COVID-19 outbreak started from a Wuhan fish market selling various animals such as rabbits, snakes, and bats [3]. COVID-19 is transmitted from human to human through contact, respiratory droplets, and fecal route [4]. Due to the rapid spread and sharp rise in mortality and morbidity, the World Health Organization declared COVID-19 as a pandemic in March 2020 [5]. As of August 2020, the World Health Organization reports that the UAE has 60,999 cases with 351 deaths [6]. Limited descriptive data are available for demographics, presenting characteristics and outcomes of the UAE population's hospitalized COVID-19 patients.

Patients with COVID-19 usually present with fever, myalgias, nonproductive cough, and progressive shortness of breath. In moderate to severe cases, signs of organ dysfunction, such as acute respiratory distress syndrome (ARDS), acute kidney injury, pulmonary edema, myocarditis, septic shock, and deaths, can occur [7]. Most COVID-19 studies [8] conclude that old age and coexisting illnesses are mortality determinants. We do not agree with this conclusion as our clinical observation suggests otherwise due to predominantly young patients and the United Arab Emirates [9]. Moreover, we believe other studies might not have adjusted for confounders affecting outcomes. We aimed to answer this question by including many clinical variables affecting outcome in our statistical analysis.

\section{Objectives}

We aimed to study the attributes of severe and fatal disease in the unique UAE population and analyze survival determinants in this cohort. Our outcomes of interest were mortality (survival) and length of stay in the ICU and hospital.

\section{Methods}

This study is a retrospective, single-center study of all patients' electronic medical records (EMR) with proven COVID-19 pneumonia admitted to Dubai Hospital, United Arab Emirates. We performed detailed statistical subgroup analysis, which may help clinicians, researchers, and authorities optimize future management of COVID-19 pneumonia.

Mortality and Survival Profiles of COVID-19 Patients in Dubai Hospital
We created a list of clinical parameters for our study and extracted the following data from Dubai Hospital EMR system. We included all admitted patients with pneumonia who are PCR-positive for COVID-19 between January 1,2020 and June 30, 2020 and excluded all patients $<18$ years. Demographics data included recent travel history, clinical signs, and symptoms on admission. We collected the following laboratory data: white blood cell counts, lymphocyte counts, platelets count, blood chemistries, renal indices, coagulation profile, liver function tests, inflammatory and disease markers including C-reactive protein (CRP), ferritin, procalcitonin, lactate dehydrogenase, creatinine kinase, and D-dimers. Daily levels of important markers affecting clinical decision-making were also recorded to collect a detailed daily profile of average D-dimers, CRP, ferritin, and procalcitonin.

Repeated nasopharyngeal or tracheal swab results have recorded the evidence of seroconversion as per protocol. We recorded the number of days to conversion and the number of swabs performed. Other culture studies include blood cultures. Treatment profiles were recorded to use chloroquine, antivirals, antibacterials, immunosuppressives, plasma therapy, and steroids. We also recorded the use of invasive mechanical ventilation, recruitment maneuvers, prone positioning, continuous renal replacement therapy (CRRT), and extracorporeal membrane oxygenation.

Definitions, clinical characteristics, and signs and symptoms were categorized as present or absent, for example, fever and cough. Laboratory characteristics were categorized in some cases (lymphopenia for count $<1,500$ cells per cubic milliliters) or continuous numerical variables (WBC, CRP, ferritin, D-dimer, and procalcitonin). Diagnosis of diseases, ARDS, pneumonia, acute kidney failure, acute heart failure, and rhabdomyolysis was extracted from EMR as charted by clinicians taking care of the patients. All laboratory tests included PCR confirmation of COVID-19 and were performed at the Dubai Hospital Laboratory.

\section{Statistical Analyses}

Continuous variables were expressed as medians with interquartile range when not normal distribution and mean \pm standard deviation for normal distribution. Categorical variables were presented as counts and percentages. Patients who survived were compared to patients who did not survive. Categorical variables were compared by the $\chi^{2}$ test, while continuous outcome variables were evaluated by the Mann-Whitney test. Subgroup analyses were also performed: critical versus noncritical, mechanically ventilated versus not ventilated, and different treatment groups (anticoagulants, chloroquine, steroids, and anticoagulation). Multiple logistic regression analyses were performed to determine survival predictors. First, univariate analysis was conducted, and then those variables that were found to be significant were included in the final logistic regression analysis. All analyses were performed with SPSS version 26 (IBM Corp., Armonk, NY, USA).

\section{Results}

Demographic and clinical characteristics of our study sample $(N, 391)$ with COVID-19 pneumonia who were admitted to Dubai Hospital are shown in Tables 1-2. Only 19 patients (4.8\%) were UAE nationals, while 372 
Table 1. Sample characteristics (categorical variables)

\begin{tabular}{|c|c|c|c|c|}
\hline \multirow[t]{2}{*}{ Clinical feature } & \multirow{2}{*}{$\begin{array}{l}\text { All patients, } n(\%) \\
(N=391)\end{array}$} & \multicolumn{2}{|l|}{ Mortality } & \multirow[t]{2}{*}{$p$ value* } \\
\hline & & died $=119$ & alive $=272$ & \\
\hline Female & $65(16.6)$ & $13(10.9)$ & $52(19.1)$ & 0.045 \\
\hline Expatriates & $379(96.9)$ & $119(100)$ & $260(95.5)$ & 0.020 \\
\hline Fever & $333(85.1)$ & $105(88.2)$ & $228(83.8)$ & 0.259 \\
\hline Cough & $308(78.7)$ & $98(82.3)$ & $212(77.9)$ & 0.322 \\
\hline Dyspnea & $279(71.3)$ & $94(78.9)$ & $185(68)$ & 0.027 \\
\hline Gastric symptoms & $54(13.8)$ & $15(12.6)$ & $39(14.3)$ & 0.648 \\
\hline Diabetes & $158(40.4)$ & $48(40.3)$ & $110(40.4)$ & 0.984 \\
\hline Hypertension & $89(22.7)$ & $31(26)$ & $58(21.3)$ & 0.305 \\
\hline CAD & $27(6.9)$ & $12(10)$ & $15(5.5)$ & 0.101 \\
\hline Renal disease & $50(12.7)$ & $21(17.6)$ & $29(10.6)$ & 0.057 \\
\hline Immunodeficiency & $19(4.8)$ & $7(5.8)$ & $12(4.4)$ & 0.534 \\
\hline History of travel & $12(3)$ & 0 & $12(4.4)$ & 0.020 \\
\hline Known exposure & $44(11.2)$ & $11(9.2)$ & $33(12.1)$ & 0.406 \\
\hline \multicolumn{5}{|c|}{ Clinical variables, $n(\%)$} \\
\hline Inpatient fever & $300(76.7)$ & $101(84.8)$ & $199(73.1)$ & 0.012 \\
\hline Tachycardia & $259(66.2)$ & $100(84)$ & $159(58.4)$ & $<0.001$ \\
\hline Hypotension & $129(32.9)$ & $70(58.8)$ & $59(21.6)$ & $<0.001$ \\
\hline Hypoxia & $255(65.2)$ & $107(89.9)$ & $161(59.1)$ & $<0.001$ \\
\hline Mechanical vent & $200(51.1)$ & $104(87.3)$ & $96(35.2)$ & $<0.001$ \\
\hline Vasopressors & $186(47.5)$ & $102(85.7)$ & $84(30.8)$ & $<0.001$ \\
\hline CRRT & $71(18.1)$ & $50(42)$ & $21(7.7)$ & $<0.001$ \\
\hline Bacterial infection & $121(30.9)$ & $64(53.7)$ & $57(20.9)$ & $<0.001$ \\
\hline Bacteremia & $94(24)$ & $53(44.5)$ & $41(15)$ & $<0.001$ \\
\hline Lymphopenia & $185(47.3)$ & $66(55.4)$ & $119(43.7)$ & 0.033 \\
\hline \multicolumn{5}{|l|}{ Treatment, $n(\%)$} \\
\hline Chloroquine & $324(82.8)$ & $99(83.1)$ & $225(82.7)$ & 0.909 \\
\hline Lopinavir/ritonavir & $107(27.3)$ & $51(42.9)$ & $56(20.5)$ & $<0.001$ \\
\hline Favipiravir & $301(76.9)$ & $85(71.4)$ & $216(79.4)$ & 0.084 \\
\hline Steroids & $226(57.8)$ & $87(73.1)$ & $139(51.1)$ & $<0.001$ \\
\hline Tocilizumab & $45(11.5)$ & $22(18.4)$ & $23(8.4)$ & 0.004 \\
\hline ECMO & $12(3)$ & $7(5.8)$ & $5(1.8)$ & 0.033 \\
\hline Sedatives & $208(53.1)$ & $113(94.9)$ & $95(34.9)$ & $<0.001$ \\
\hline Narcotics & $178(45.5)$ & $94(78.9)$ & $84(30.8)$ & $<0.001$ \\
\hline Paralytics & $199(50.8)$ & $104(87.3)$ & $95(34.9)$ & $<0.001$ \\
\hline Anticoagulation & $350(89.5)$ & $114(95.7)$ & $236(86.7)$ & 0.007 \\
\hline GI prophylaxis & $352(90)$ & $113(94.9)$ & $239(87.8)$ & 0.031 \\
\hline
\end{tabular}

CAD, coronary artery disease; CRRT, continuous renal replacement therapy; ECMO, extracorporeal membrane oxygenation. ${ }^{*} \chi^{2}$ to compare categorical variables.

patients (95.2\%) were expatriates. The patients' median age was 48 (interquartile range, $40-56$ ) years; $22 \%$ of the patients were younger than 40 years. Only $16.6 \%$ were female. Eighty-five percent of the patients reported a fever history at home, and 77.4 percent had a fever on admission. The most common symptom was cough (78.7\%), and the least common were gastrointestinal complaints (13.8\%). Among the overall population, $40 \%$ had diabetes, $23 \%$ had hypertension, and only $6 \%$ had heart disease.
Patients admitted to the ICU were older than those not admitted in the ICU $(49.6 \pm 10.9$ vs. $46.7 \pm 12.7 p=0.04)$. Moreover, diabetes ( 43 vs. $39 \%$ ) and hypertension (25 vs. $21 \%$ ) were more common among patients admitted to the ICU than those not admitted to the ICU. Among patients admitted to the ICU, $85 \%$ required invasive mechanical ventilation, $78 \%$ required vasopressors, and $30 \%$ required CRRT on admission. In contrast, none needed in the group were admitted to the medical ward. 
Table 2. Sample characteristics (continuous variables)

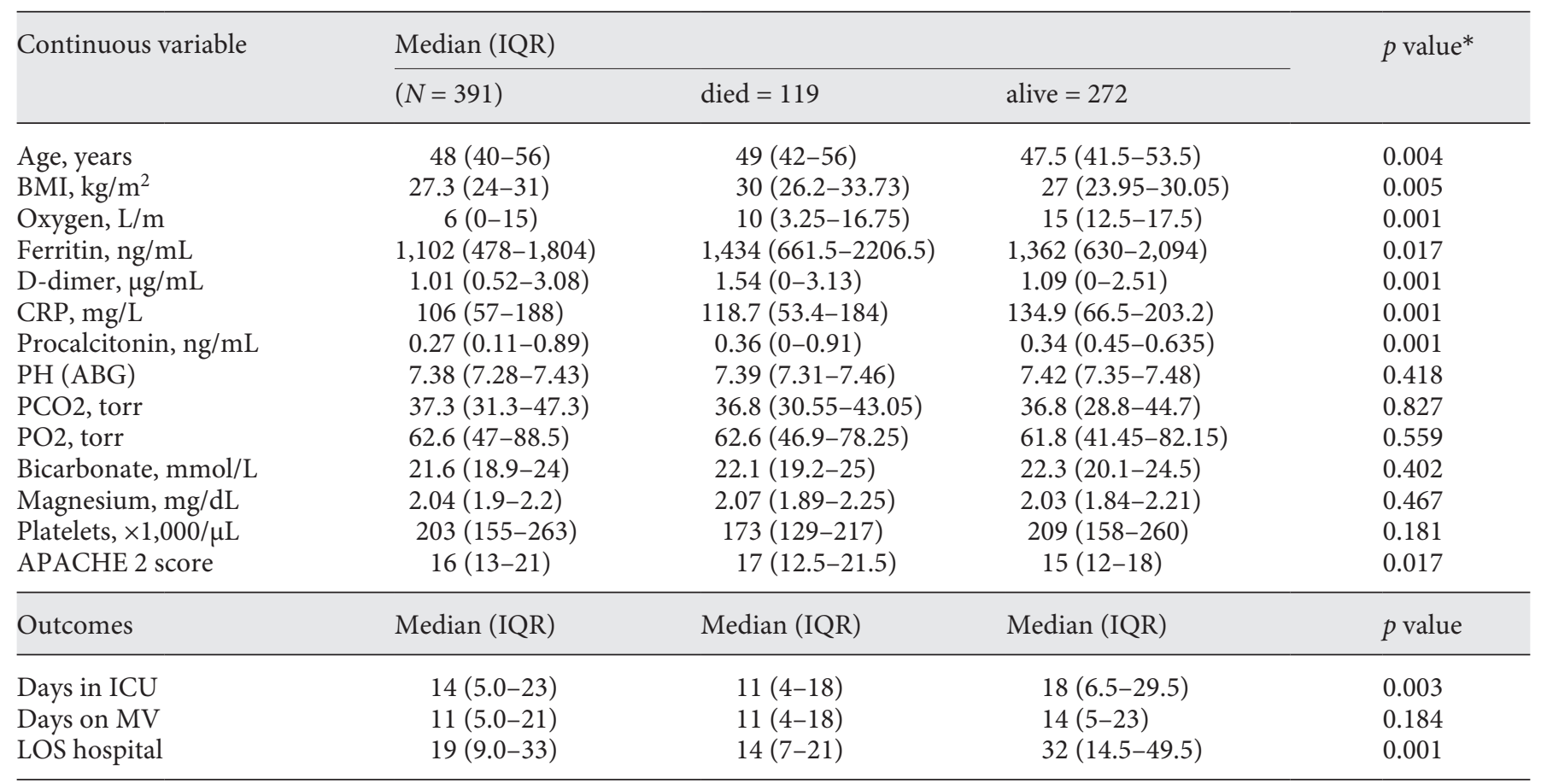

CRP, C-reactive protein; APACHE, Acute Physiology and Chronic Health Evaluation. * Mann-Whitney U test is used to compare variables.

On admission, lymphocytopenia was present in $47.3 \%$ and thrombocytopenia in $13.8 \%$ of hospitalized patients. Most of the patients (>95\%) had elevated levels of CRP and D-dimers (79\%). Multiple swabs for COVID-19 PCR were performed (mean $=5$ swabs). Patients took a long time for seroconversion as documented by COVID-19 PCR swab for ICU patients compared to non-ICU patients ( 15 vs. 13 days $p=0.01$ ).

Among the patients admitted to the ICU, extracorporeal membrane oxygenation was utilized in 13 patients, among whom 6 of them survived (46\%); MV is required in $85 \%$, vasopressors were required in $79 \%$, sedation in $88 \%$, and muscle paralysis in $84 \%$ patients. Survivors have less proportion on sedatives $(p=0.01)$. Paralytics use was not statistically different between survivors and nonsurvivors. CRRT was utilized in $30 \%$ of patients for renal impairment. Survivors were less likely to be treated with CRRT than nonsurvivors ( 18 vs. $42 \% p=0.01$ ). More than half of the patients had evidence of secondary bacterial infections during ICU stay. There was no significant difference in the prevalence of bacterial infections between survivors and nonsurvivors. However, bacteremia was more common in nonsurvivors than survivors (44.5 vs.
$15 \%, p$ 0.022). Only $12 \%$ underwent tracheostomy; $80 \%$ of those who had tracheostomy survived, compared to $44 \%$ who did not have a tracheostomy. Comparison of treatment profiles of survivors versus nonsurvivors is presented in Tables 1-2.

Mortality was $30 \%$ for the total sample $(N 391)$ and $50 \%$ for those admitted to the ICU (N 240). Among the mechanically ventilated $(N=200)$ patients, more than half (52\%) died.

The median duration of hospitalization was 19 (IQR 9-33) days, ICU stays 14 (IQR 5-23) days, and ventilator days 11 (IQR 5-21) days. Analysis of outcome measures of LOSICU, LOSH, and ventilator days by the MannWhitney test showed that survivors spent more days in the ICU (median [IQR] 18 [6.5-29.5] vs. 11 [4-18], p value 0.003 ) and in the hospital (32 [14.5-49.5] vs. 14 [7-21], $p$ value 0.001$)$ than nonsurvivors. Ventilator days were not statistically different between the 2 groups (14 [5-23] vs. 11 [4-18], $p$ value $=0.18$ ). Multiple logistic regression analysis determined age, inpatient fever, use of oxygen, mechanical ventilation, and steroids as predictors of mortality (Table 3). We constructed a Kaplan-Meier plot for the impact of steroids on LOS (Fig. 1). 
Table 3. Multiple logistic regression - predictors of mortality

\begin{tabular}{|c|c|c|c|c|c|}
\hline \multirow[t]{2}{*}{ Variable } & \multirow[t]{2}{*}{ B } & \multirow[t]{2}{*}{$p$ value } & \multirow{2}{*}{$\begin{array}{l}\text { Odds } \\
\text { ratio }\end{array}$} & \multicolumn{2}{|c|}{$95 \%$ CI for odds ratio } \\
\hline & & & & lower & upper \\
\hline Female gender & 0.096 & 0.913 & 1.100 & 0.197 & 6.131 \\
\hline Dyspnea & 0.158 & 0.816 & 1.171 & 0.310 & 4.423 \\
\hline Inpatient fever & 2.070 & 0.039 & 7.922 & 1.115 & 56.289 \\
\hline Tachycardia & 0.175 & 0.793 & 1.192 & 0.323 & 4.399 \\
\hline Hypotension & -0.312 & 0.587 & 0.732 & 0.238 & 2.254 \\
\hline Ventilation & 2.530 & 0.027 & 12.548 & 1.342 & 117.306 \\
\hline Vasopressors & -1.464 & 0.198 & 0.231 & 0.025 & 2.148 \\
\hline Dialysis & -0.086 & 0.880 & 0.917 & 0.299 & 2.815 \\
\hline Bacterial infection & 0.189 & 0.785 & 1.208 & 0.311 & 4.691 \\
\hline Bacteremia & 0.210 & 0.765 & 1.234 & 0.312 & 4.877 \\
\hline Lymphopenia & -0.284 & 0.569 & 0.753 & 0.284 & 1.998 \\
\hline Lopinavir/ritonavir & -0.708 & 0.141 & 0.493 & 0.192 & 1.264 \\
\hline Steroids & 2.229 & 0.011 & 9.293 & 1.658 & 52.083 \\
\hline Tocilizumab & -1.113 & 0.061 & 0.328 & 0.103 & 1.051 \\
\hline ECMO & 0.277 & 0.782 & 1.320 & 0.185 & 9.408 \\
\hline Sedatives & -2.484 & 0.332 & 0.083 & 0.001 & 12.606 \\
\hline Narcotics & 0.333 & 0.663 & 1.395 & 0.311 & 6.256 \\
\hline Paralytics & -0.208 & 0.923 & 0.812 & 0.012 & 54.282 \\
\hline Anticoagulation & -2.697 & 0.252 & 0.067 & 0.001 & 6.786 \\
\hline GI prophylaxis & -0.944 & 0.551 & 0.389 & 0.017 & 8.705 \\
\hline Age, years & 0.054 & 0.038 & 1.056 & 1.003 & 1.112 \\
\hline BMI & -0.003 & 0.863 & 0.997 & 0.959 & 1.036 \\
\hline Oxygen & -0.099 & 0.019 & 0.906 & 0.834 & 0.984 \\
\hline Ferritin & 0.000 & 0.134 & 1.000 & 1.000 & 1.001 \\
\hline D-dimer & -0.006 & 0.815 & 0.994 & 0.945 & 1.046 \\
\hline CRP & 0.000 & 0.890 & 1.000 & 0.995 & 1.006 \\
\hline Procalcitonin & -0.014 & 0.799 & 0.986 & 0.884 & 1.100 \\
\hline APACHE-2 score & 0.004 & 0.923 & 1.004 & 0.923 & 1.092 \\
\hline
\end{tabular}

CRP, C-reactive protein; APACHE, Acute Physiology and Chronic Health Evaluation; ECMO, extracorporeal membrane oxygenation.

\section{Discussion}

Our sample predominantly includes young, male expatriates of the UAE, admitted in Dubai Hospital with COVID-19 pneumonia and with pre-existing medical illnesses. Our median sample age was 48 years, which is different from the Italian population ( $>70$ years) and American patients (67 years) $[10,11]$. Obesity was a predictor of mortality in American patients [11], while our sample's mean BMI was 27, and it was not a predictor of mortality. Percent of expatriates was not known for the American or European population, while our sample includes 95\% expatriates.

Our study's survival rate was $70 \%$, which is like recent studies [12], and higher than that in earlier studies with insufficient data about follow-up [1, 13, 14]. Survival among those requiring invasive mechanical ventilation

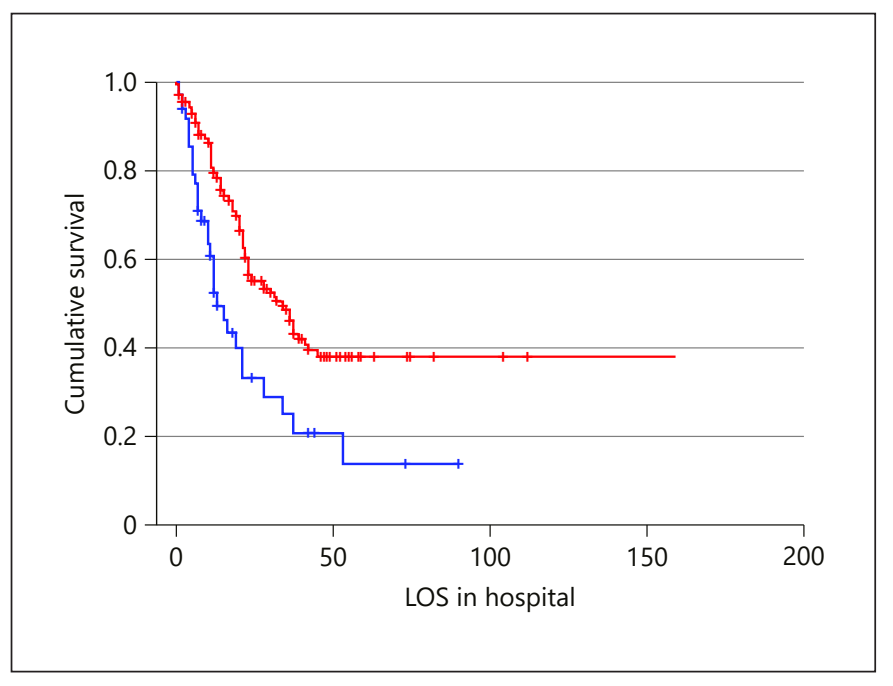

Fig. 1. Kaplan Meier plot for effect of steroids on length of stay. 
was $48 \%$, which is less than that other studies $[9,15]$. One reason could be that previous studies had incomplete follow-up data as they were published with the ongoing pandemic.

Among the mechanically ventilated patients, those treated with steroids had better survival than those not treated with steroids ( $52 \mathrm{vs.} 36 \%, p=0.04$ ). Similar results were reported by the RECOVERY trial [16]. Among patients with nonsevere disease and those not requiring mechanical ventilation, treatment with steroids adversely affected survival (61 vs. $79 \%, p=0.01$ ). A recent trial from Brazil did not detect any survival benefit from steroids [15]. Treatment with steroids has been documented to be a predictor of mortality in mild disease [16].

Steroid dosages and duration was variable - hydrocortisone from 200 to $400 \mathrm{mg} /$ day or methylprednisolone $40-80 \mathrm{mg} /$ day or dexamethasone $12-32 \mathrm{mg} /$ day. Duration ranges from single dose on admission to more than 2 weeks course. The choice of steroid, dose, and duration was at the discretion of the treating physician.

Treatment with chloroquine was similar between survivors and nonsurvivors ( 82.7 vs. $83.1 \%, p=0.6)$, which are like results reported by Cavalcanti et al. [17]. However, survivors were less frequently treated with lopinavir-ritonavir (20.5 vs. $42.9 \%, p=0.01)$. Among mechanically ventilated patients, survival with lopinavir-ritonavir was also worse ( 40 vs. $55 \%, p=0.04$ ). A recent trial also showed no survival advantage with lopinavir-ritonavir treatment [18]. Prior studies [19] found multiple factors predicting mortality, such as age and comorbid conditions, which we did not predict; most likely, our population and methodology were different. We also adjusted for numerous confounding factors known to determine mortality. Arbitrary exclusion of many such confounders in our hypothetical model also provided us similar predictors of mortality; therefore, the omission of confounding factors may have played a role in other studies.

Patients requiring vasopressors and CRRT reflect the severity of organ dysfunction; therefore, finding these factors as determinants of survival for COVID-19 pneumonia is no surprise. Similar results were documented by other studies [20]. CRP, ferritin, and D-dimers were significantly lower in patients who survived, and they were not predictors of survival, as suggested by other studies [20]. This difference in results is most likely due to differences in methodology, statistical modeling, and different inclusion of confounding factors.

Our study has the following weaknesses. This study is a retrospective, single-center, community hospital study; therefore, results may not apply to other populations. To our knowledge, no research has documented the clinical profile of patients who had died from COVID-19 pneumonia in the Middle East with the inclusion of all relevant clinical predictors as we did. Other studies found many predictors of survival, including steroids. Well-designed, prospective, multicenter trials with large sample size, preferably with the inclusion of all critical clinical factors affecting survival, are urgently needed to determine a better management strategy.

\section{Conclusion}

Patients affected by COVID-19 were predominantly young, expatriate males with pre-existing conditions of diabetes and hypertension. Outcomes with chloroquine, antivirals, and anticoagulation were not different between survivors and nonsurvivors. The majority admitted suffered severe ARDS requiring invasive mechanical ventilation and renal replacement therapy. Markers of disease activity, ferritin, CRP, and D-dimers were higher in patients who died. Multiple logistic regression analyses determined steroid treatment to be a predictor of mortality.

\section{Statement of Ethics}

Ethical approval was provided by DSREC-07/2020_10/approved on July 13, 2020 (according to Local Ethical Committee guidelines, for retrospective medical records chart review observational studies; no consent from patients are required).

\section{Conflict of Interest Statement}

The authors have no conflicts of interest to declare.

\section{Funding Sources}

The authors did not receive any funding.

\section{Author Contributions}

R.N.: conceived the research idea, proposal writing, data collection, data analysis, and manuscript writing. I.A. and A.E.: conceived the idea, proposal writing, and review of final manuscript. C.D. and N.A.: idea conception and data collection. M.K.: data collection. M.A., M.J., A.N., S.T., S.S., M.H., M.K., I.B., A.E., L.S., S.K., and R.A.: idea conception and data collection. 


\section{References}

1 Guan W-j, Ni Z-y, Hu Y, Liang W-h, Ou C-q, $\mathrm{He} J-\mathrm{x}$, et al. Clinical characteristics of coronavirus disease 2019 in China. N Engl J Med. 2020;382(18):1708-20.

2 Mehta P, McAuley DF, Brown M, Sanchez E, Tattersall RS, Manson JJ; HLH Across Specialty Collaboration. COVID-19: consider cytokine storm syndromes and immunosuppression. Lancet. 2020;395(10229):1033-4.

3 Bai Y, Yao L, Wei T, Tian F, Jin DY, Chen L, et al. Presumed asymptomatic carrier transmission of COVID-19. JAMA. 2020;323(14): 1406-7.14

4 Wang D, Hu B, Hu C, Zhu F, Liu X, Zhang J, et al. Clinical characteristics of 138 hospitalized patients with 2019 novel coronavirus-infected pneumonia in Wuhan, China. JAMA. 2020;323(11):1061-9.

5 Wang C, Horby PW, Hayden FG, Gao GF. A novel coronavirus outbreak of global health concern. Lancet. 2020;395(10223):470-3.

6 World Health Organization. Coronavirus disease (COVID-2019) situation reports.

7 Huang C, Wang Y, Li X, Ren L, Zhao J, Hu Y, et al. Clinical features of patients infected with 2019 novel coronavirus in Wuhan, China. Lancet. 2020;395(10223):497-506.

8 Bonanad C, García-Blas S, Tarazona-Santabalbina F, Sanchis J, Bertomeu-González V, Fácila L, et al. The effect of age on mortality in patients with COVID-19: a meta-analysis with 611,583 subjects. Hypertension. 962(23): 1366.
9 Richardson S, Hirsch JS, Narasimhan M, Crawford JM, McGinn T, Davidson KW, et al. Presenting characteristics, comorbidities, and outcomes among 5700 patients hospitalized with COVID-19 in the New York City area. JAMA. 2020

10 Boccia S, Ricciardi W, Ioannidis JPA. What other countries can learn from Italy during the COVID-19 pandemic. JAMA Intern Med. 2020;180(7):927-8.

11 Palaiodimos L, Kokkinidis DG, Li W, Karamanis D, Ognibene J, Arora S, et al. Severe obesity, increasing age and male sex are independently associated with worse in-hospital outcomes, and higher in-hospital mortality, in a cohort of patients with COVID-19 in the Bronx, New York. Metabolism. 2020;108: 154262.

12 Weiss P, Murdoch DR. Clinical course and mortality risk of severe COVID-19. Lancet. 2020;395(10229):1014-5.

13 Chen N, Zhou M, Dong X, Qu J, Gong F, Han $\mathrm{Y}$, et al. Epidemiological and clinical characteristics of 99 cases of 2019 novel coronavirus pneumonia in Wuhan, China: a descriptive study. Lancet. 2020;395:507-13.

14 Huang C, Wang Y, Li X, Ren L, Zhao J, Hu Y, et al. Clinical features of patients infected with 2019 novel coronavirus in Wuhan, China. Lancet. 2020;395:497-506.
15 Jeronimo CMP, Farias MEL, Val FFA, Sampaio VS, Alexandre MAA, Melo GC, et al. Methylprednisolone as adjunctive therapy for patients hospitalized with COVID-19 (MetCOVID): a randomised, double-blind, phase IIb, placebo-controlled trial. Clin Infect Dis. 2020.

16 RECOVERY Collaborative Group. Dexamethasone in hospitalized patients with COVID-19: preliminary report. N Engl J Med. 2020.

17 Cavalcanti AB, Zampieri FG, Rosa RG, Azevedo LCP, Veiga VC, Avezum A, et al. Hydroxychloroquine with or without Azithromycin in mild-to-moderate COVID-19. N Engl J Med. 2020. 383:2041-52.

18 Cao B, Wang Y, Wen D, Wen L, Wang J, Fan $\mathrm{G}$, et al. A trial of lopinavir-ritonavir in adults hospitalized with severe COVID-19. N Engl J Med. 2020. 382:1787-99.

19 Petrilli CM, Jones SA, Yang J, Rajagopalan $\mathrm{H}$, O'Donnell L, Chernyak Y, et al. Factors associated with hospitalization and critical illness among 4,103 patients with COVID-19 disease in New York City. MedRxiv. 2020.

20 Cummings MJ, Baldwin MR, Abrams D, Jacobson SD, Meyer BJ, Balough EM, et al. Epidemiology, clinical course, and outcomes of critically ill adults with COVID-19 in New York City: a prospective cohort study. Lancet. 2020. 395(10239):1763-70. 